\title{
TERAPIA COGNITIVO-COMPORTAMENTAL PARA O TRATAMENTO DO TRANSTORNO DE ANSIEDADE SOCIAL: UMA REVISÃO SISTEMÁTICA
}

DOI: $10.22289 / 2446-922 X . V 2 E E A 4$

\author{
Érica Ires Fernandes ${ }^{4}$ \\ Résia Silva de Morais
}

\section{RESUMO}

O Transtorno de Ansiedade Social é um distúrbio caracterizado pela incidência de medo exacerbado e persistente do indivíduo ao se expor em circunstâncias sociais, causando diferentes impactos em sua vida. No presente estudo busca-se descrever técnicas e resultados referentes ao tratamento do Transtorno da Ansiedade Social, por meio da Terapia Cognitivo-comportamental. Realizou-se uma revisão sistemática da literatura. Para o levantamento dos artigos foram feitas buscas eletrônicas em bases de dados a saber: Lilacs, Pepsic, PsycINFO, Scielo, Springerlink e ScienceDirect - Elsevier. Observou-se que a Terapia Cognitivo-comportamental (TCC) pode contribuir para o tratamento do Transtorno de Ansiedade Social (TAS), por meio de diferentes técnicas, como exposição ao vivo, reestruturação cognitiva, relaxamento e treino das habilidades sociais. Conclui-se que, as técnicas da TCC apresentam-se eficazes para o tratamento do TAS, podendo estas, contribuírem para se obter resultados eficazes para o tratamento, de maneira que o paciente desenvolva as suas atividades sociais e alcance maior qualidade de vida.

Palavras-chave: Enfrentamento; Fobia;Transtorno de Ansiedade Social; Terapia CognitivoComportamental; Técnicas.

\section{ABSTRACT}

Social Anxiety Disorder is a disorder characterized by the incidence of exaggerated and persistent fear of the individual to be exposed in social circumstances, causing different impacts on your life. In the present study seeks to describe techniques and results for the treatment of Social Anxiety Disorder, through cognitive-behavioral therapy. We conducted a systematic review of the literature. For the survey of articles electronic searches were made in databases such as Lilacs, Pepsic; Psycinfo, Scielo, and SpringerLink and ScienceDirect Elsevier. It was observed that cognitive-behavioral therapy can contribute to the treatment of Social Anxiety Disorder, by using different techniques, such as in vivo exposure, cognitive restructuring, relaxation training and social skills. In conclusion, the relevant technical cognitive-behavioral therapy have proven effective for the treatment of Social Anxiety Disorder and may these, contribute to achieve effective results in the treatment so that the patient develops its social activities and can get higher quality of life.

Kewords: Cognitive behavioral therapy; Coping; Phobia; Social Anxiety Disorder; Techniques.

${ }^{4}$ Endereço eletrônico de contato: ericadiskbel@hotmail.com

Outubro, 2016:2(Edição Especial):41-53. 


\section{INTRODUÇÃO}

O Transtorno de Ansiedade Social, também chamado de Fobia Social, refere-se a um distúrbio caracterizado pela incidência de medo exasperado e persistente do indivíduo, ao se expor em situações sociais, gerando intensa ansiedade e sofrimento. (1)

Considerada como um dos transtornos mentais mais prevalentes na população geral, o Transtorno de Ansiedade Social, causa diferentes impactos na vida do indivíduo, pois, além de comprometer o seu desenvolvimento cognitivo, a sua tendência é ficar à margem da sociedade, visto que, a fobia o afasta da inserção social, da atividade laborativa e do desenvolvimento cultural. ${ }^{(2)}$

Na classificação do DSM - 5 (critério diagnóstico 300.23), a fobia social se caracteriza pela presença de medo ou ansiedade acentuados acerca de uma ou mais situações sociais, em que o indivíduo é exposto a possível avaliação de outras pessoas em diferentes formas de interações sociais, como conversar com alguém que não seja da família, ser observado ao comer ou beber, e se colocar em situações de desempenho diante dos outros, como proferir palestras. ${ }^{(1)}$

O Transtorno de Ansiedade Social é prevalente na população geral e causa diferentes impactos na vida do indivíduo, incapacitando-a para o convívio social e a Terapia Cognitivocomportamental pode oferecer contribuições para o tratamento do Transtorno de Ansiedade Social. (2)

O modelo cognitivo comportamental pode ser sugerido, para a formulação de novos modelos etiológicos e para o tratamento de transtornos mentais procedentes dos transtornos de ansiedade, como transtorno obsessivo-compulsivo, ansiedade generalizada, transtorno do pânico e a ansiedade social. A eficácia da TCC vem sendo recomendada para os quadros depressivos unipolares, transtorno do pânico com e sem agorafobia, fobia social, transtorno de estresse pós-traumático, transtorno obsessivo-compulsivo, esquizofrenia e bulimia nervosa. ${ }^{(3)}$

Por meio das técnicas de TCC, o paciente com TAS tem condições de aprender habilidades cognitivas e comportamentais imprescindíveis para melhorar o seu desempenho interpessoal e modificar os seus pensamentos distorcidos. ${ }^{(4)} \mathrm{A}$ realidade ora referida sugere a necessidade de se conhecer, em meio às diferentes técnicas que a TCC dispõe, algumas capazes de proporcionar maior eficiência para reduzir o TAS, como técnicas de relaxamento, treino de habilidades sociais, técnicas de reestruturação cognitiva, exposição ao vivo, TCC em grupos, TCC via internet e a psicoeducação.

Outubro, 2016:2(Edição Especial):41-53. 
Pesquisas acadêmicas como um artigo científico devem apresentar uma relevância científica e social. ${ }^{(5)}$ No caso da pesquisa em tela, cabe ressaltar primeiramente que, muitos males que acometem a sociedade, estão ligados a distúrbios de ordem comportamental, dentre eles, enfatiza-se o Transtorno de Ansiedade Social, cujo problema pela sua importância, requer muitos estudos que visem contribuir para o entendimento e formulação de propostas de tratamento eficazes. Eis, então, a relevância científica deste trabalho, que se preocupa em apreciar métodos, técnicas e resultados referentes ao tratamento do Transtorno da Ansiedade Social, por meio da Terapia Cognitivo-comportamental, a partir de uma revisão sistemática.

A revisão sistemática se trata de um estudo secundário, cujo objetivo é reunir estudos similares, de modo a avaliá-los criticamente em sua metodologia. "Por sintetizar estudos primários semelhantes e de boa qualidade é considerada o melhor nível de evidência para tomadas de decisões em questões sobre terapêutica." (6)

Além disso o estudo se caracterizou como uma revisão sistemática qualitativa descritiva. A pesquisa qualitativa propicia melhor visão e apreensão do contexto do problema estudado. (7) Descritiva, pois pesquisas descritivas têm o objetivo de fazer a descrição das peculiaridades de uma população, fenômeno ou de uma experiência. ${ }^{(5)}$

\section{MATERIAL E MÉTODOS}

O material utilizado procede de textos das bases de dados on-line e o método é o da seleção, análise e tratamento das informações. Os descritores de busca foram: Transtorno de Ansiedade Social, Terapia Cognitivo-comportamental. Técnicas de Terapia Cognitivocomportamental. O período de publicação dos estudos consultados para confeccionar a base teórica de todo o trabalho, foi de 1998 a 2015. Mas, para realizar a análise sistemática o período de publicação dos estudos foi de 2005 a 2015. Os idiomas foram Português, Inglês e Espanhol.

Para o levantamento dos artigos, foram realizadas buscas eletrônicas em seis bases de dados, a saber: Lilacs (Literatura Latino-Americana e do Caribe em Ciências da Saúde), Pepsic (Periódicos Eletrônicos de Psicologia); PsycINFO (American Psychological Association - APA), Scielo (Scientific Electronic Library Online), Springerlink e ScienceDirect

- Elsevier. Essa seleção se justifica por serem bases reconhecidas e por serem bastante utilizadas em revisões sistemáticas da literatura nas áreas de saúde e Psicologia.

Outubro, 2016:2(Edição Especial):41-53. 
Primeiramente, foram analisados os títulos dos artigos com a finalidade de descartar referências repetidas. Posteriormente, selecionaram-se os resumos anexados e foi realizada uma leitura prévia para eleger a seleção do material necessário para as revisões. Essa análise adotou os seguintes critérios de inclusão: 1) palavras descritoras presentes no corpo do texto; 2) idioma: artigos que foram publicados na integra em Inglês, Espanhol e Português; 3) formato: foram selecionadas as referências publicadas como artigo, relato de experiência ou revisão da literatura científica e foram recusadas as referências publicadas como livro, capítulo de livro, dissertação, tese, resenha, carta ao editor e resumo; 4) foram selecionadas somente em uma base, sendo descartadas nas outras e 5) foram escolhidas apenas as referências relacionadas ao tema.

Por meio dos artigos selecionados formou-se o corpus que definiu o material de análise e a apreciação de oito dimensões de análise, a saber: (1) Título; (2) Autores/Ano de publicação; (3) País da Publicação; (4) Critérios de inclusão e exclusão/amostra; (5) Objetivo do estudo; (6) Instrumentos utilizados para avaliação e/ou intervenção; (7) Resultados; e, (8) Conclusões.

\section{RESULTADOS}

\subsection{APRECIAÇÃO QUANTITATIVA DAS REFERÊNCIAS}

A base de dados LILACS possibilitou identificar um total de 19 artigos relacionados. Mas, após a aplicação dos critérios de inclusão, foram selecionados 8 artigos. Na base PEPSIC foram encontrados 28 artigos e apenas 2 foram utilizados. Na base PSYCINFO foram encontrados apenas 13 artigos, porém 2 artigos foram selecionados. Na base SciELO foram encontradas 12 referências, 6 foram escolhidas. Na base de dados SCIENCEDIRECT ELSEVIER foram encontrados 101 artigos, sendo apenas 2 discriminados. Na base SPRINGERLINK a busca resultou em 27 referências de estudos publicados em periódicos, dos quais, apenas 1 foi selecionado. Deste modo, os dados da revisão de literatura foram tabelados, e resultaram em um total de 21 artigos. A Figura 1 apresenta o percurso de seleção dos artigos.

Outubro, 2016:2(Edição Especial):41-53. 


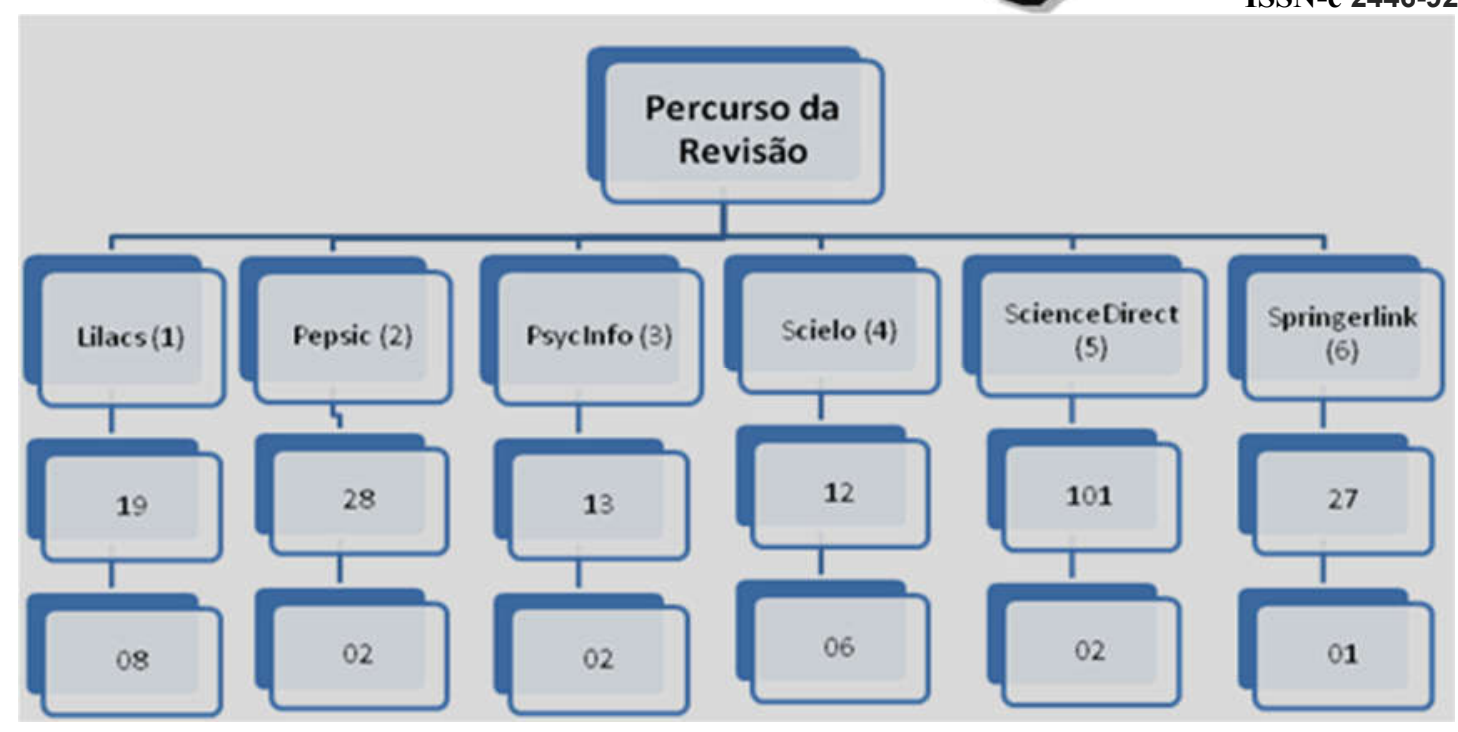

Figura 1: Organograma do percurso da Revisão.

O Quadro 1 apresenta o processo de seleção dos artigos da revisão, e o total cujas referências foram avaliadas qualitativamente seguindo algumas dimensões de análise.

Quadro 1 - Percurso de seleção dos artigos da revisão

\begin{tabular}{|l|l|l|l|}
\hline \hline Base de dados & No de referências & $\mathbf{N}^{\circ}$ de referências & $\begin{array}{l}\text { Dimensões } \\
\text { análise }\end{array}$ \\
selecionadas & (08 Dimensões)
\end{tabular}

Outubro, 2016:2(Edição Especial):41-53. 


\section{DISCUSSÃO}

\subsection{APRECIAÇÃO QUALITATIVA DAS REFERÊNCIAS}

A primeira dimensão estudada foram os Títulos com o intuito de priorizar artigos pertinentes a Terapias Cognitivo-Comportamentais para o Transtorno de Ansiedade Social que é o tema de interesse do presente estudo. De modo geral os títulos dos artigos selecionados são bem direcionados para o tema deste estudo, pois autores, como os das obras $^{(8-20)}$ anunciam explicitamente o tratamento para o TAS por meio da TCC.

A segunda dimensão foram os Autores e Ano de publicação. O processo de seleção selecionou publicações que compreendesse o período de 2005 a 2015, a fim de observar se houve modificação de propostas de técnicas associadas à TCC para o TAS, neste período, e certificou-se que ao longo de 10 anos, ao menos nos estudos analisados, observou-se uma maior difusão dos meios tecnológicos como recursos de comunicação para aplicar técnicas da TCC ao TAS.

Interessante observar também que cinco dos artigos estudados foram publicados no ano de 2008, ano este que sugere ter tido mais divulgação do tratamento de TCC para o TAS. $(8,17-23)$

Diante da terceira dimensão (País da Publicação), observa-se que a maior parte da produção científica desta revisão é oriunda do Brasil, ou seja, (13) artigos, e os demais são: (1) Buenos Aires, (1) Madrid, (2) Espanha, (1) Bogotá, (2) EUA e (1) Londres. Pode-se observar ainda que ao menos na amostra analisada, os artigos em Inglês, Português e Espanhol não revelam grandes diferenças de técnicas da TCC para a gestão da fobia social, pois os métodos utilizados fora do país são praticamente os mesmos utilizados no Brasil.

Já na quarta dimensão os critérios estudados foram de inclusão e exclusão em relação à amostra. Foram apresentados 14 casos clínicos, cujas amostras são bem diversificados, no que se refere ao sexo, idade, nível sócio econômico cultural e profissional, dentre os sujeitos estudados nestes estudos, constatam-se adolescentes, profissionais de saúde, médicos, psicólogos e estudantes universitários, o único requisito para todos estes estudos é que os sujeitos fossem portadores do Transtorno de Ansiedade Social.

Explanam-se ainda 7 estudos teóricos apresentados por autores,que tiveram como critério de inclusão, trabalhos cuja abordagem fosse voltada exclusivamente para o tratamento do Transtorno de Ansiedade Social, por meio da Terapia Cognitiva Comportamental, de modo

Outubro, 2016:2(Edição Especial):41-53. 
geral, para levantar os dados teóricos os autores consultaram bases de dados como PubMed, Medline, Psycinfo, Lilacs, Capes, Scielo, BVS Psi. (12,18,21,24-26)

A quinta dimensão estudada apresenta qual o objetivo que os artigos buscaram em presença da pesquisa realizada, e todos os estudos consultados, tanto teóricos, quanto de casos clínicos, tiveram como objetivo, estudar o tratamento do Transtorno de Ansiedade Social por meio da Terapia Cognitivo-Comportamental, compreender as técnicas de enfrentamento no tratamento e sua eficácia.

Na sexta dimensão as técnicas de Intervenção mais utilizadas pelos autores foram treino de habilidades sociais ${ }^{(21,25,11,12,16)}$, reestruturação cognitiva ${ }^{(27,21,25)}$, tarefas de casa(16), técnicas de relaxamento ${ }^{(19)}$ exposição ao vivo ${ }^{(11,14)}$, psicoterapia ${ }^{(20,27)}$, psicoeducação ${ }^{(17,26)}$, exposição sistemática ${ }^{(18,16)}$ e tratamento por realidade virtual, via computador ${ }^{(16)}$. Os Instrumentos utilizados para avaliação e/ou intervenção da TCC para o TAS, envolveram questionário epidemiológico de sintomas mentais, escala de ansiedade cognitiva, inventários de depressão, medo, ansiedade e fobia social e Diagnóstico primário de TAS (segundo o DSMIII, DSM-III-R, DSM-IV ou CID-10).

Cabe lembrar-se de que, as técnicas de reestruturação cognitiva e exposição ao vivo, foram as mais citadas nos artigos analisados, estas têm o objetivo de munir o paciente para a habilidade de identificar os pensamentos negativos. Nestas técnicas, o terapeuta e o paciente trabalham juntos, de maneira a construir uma hierarquia de circunstâncias que gerem níveis crescentes da ansiedade, colaborando para que o paciente se aproxime cada vez mais do nível de ansiedade que mais teme ${ }^{(28)}$.

Diversos autores asseguram que as técnicas de reestruturação cognitiva contribuem efetivamente para municiar ao paciente a habilidade de identificar os pensamentos negativos, sendo esta técnica de suma importância para compor os tratamento do TAS. (11,12,14,17,18,21,2427)

O treino de habilidades sociais envolve a modelagem pelo terapeuta, ensaio comportamental, funcionamento social junto às atividades de casa. $(11,12,16,21,25)$

As técnicas de relaxamento visam controlar sintomas fisiológicos como sudorese, tontura e hiperventilação, antes e durante a circunstância temida, a fim de facilitar à exposição do paciente as ocasiões por ele temidas. ${ }^{(19)}$

A TCC associada à evitação de comportamentos de segurança e ao treino de habilidades sociais evidencia prerrogativa adicional se comparada ao modelo clássico. Dentre as técnicas comportamentais, outras estratégias eficazes, são o treino em atividade de

Outubro, 2016:2(Edição Especial):41-53. 
concentração e a exposição. O tratamento individualizado é avaliado mais eficaz do que em grupo, e o modelo proposto com sessões semanais foi mais eficiente do que sessões quinzenais. Comprovam-se eficácia do tratamento do TAS, tanto por meio de técnicas cognitivas, quanto por meio das técnicas comportamentais que podem ser convencionais e/ou inovadoras. As técnicas via computador são eficazes para o tratamento do TAS, especialmente as que preservam o contato com o terapeuta. (16)

Neste estado ainda explica que, não há um consenso referente à eficácia da associação da terapia cognitiva às técnicas comportamentais. Mas, técnicas, como tratamento mediante realidade virtual, atividade de casa por meio do computador e autoterapia com assistência terapêutica evidenciaram ser tão eficazes quanto às técnicas aplicadas na terapia cognitivo-comportamental (TCC) tradicional. ${ }^{(16)}$

Já a sétima dimensão, analisou os resultados: as técnicas da TCC apresentaram melhoras superiores, clara redução nos níveis gerais de ansiedade, tanto emocionais quanto fisiológicas e as expectativas do paciente sobre as relações sociais, mudaram consideravelmente, reduzindo-se assim a sua atividade relacionada ansiedade social. Além disso, os tratamentos de terapia cognitiva originalmente desenvolvidos para os adultos podem ser utilizados da mesma maneira com crianças e adolescentes. ${ }^{(8-27,28,29)}$

Por fim, a oitava dimensão levou em conta a conclusão dos dados: a melhor escolha para o tratamento de adultos é a associação da psicoterapia cognitivo-comportamental com a farmacologia. ${ }^{(20,25)}$

Os Estudos de Utilização de Medicamentos representam apoio decisivo na elaboração de políticas governamentais na área de saúde e compreendem diferentes temas de investigações relativos ao uso de medicamentos. Com estes estudos é possível compreender a demanda do uso de medicamentos e com isso verificar possibilidades de ocorrências de abuso, mau uso, uso incorreto de medicamentos e até mesmo o descumprimento da terapia. ${ }^{(30)}$

Baseado nas afirmativas deste autor se fazem necessários Estudos de Utilização de Medicamentos em cada região para assim efetuar estimativas da demanda e do perfil de comercialização de um determinado medicamento. ${ }^{(31)}$

Estudos corroboram que a TCC é eficaz para o TAS, tanto para o tratamento de jovens como de adultos. $(11,21,23,29)$

A terapia cognitivo-comportamental é considerada a forma de psicoterapia mais estudada para o TAS. ${ }^{(24,12)}$

Outubro, 2016:2(Edição Especial):41-53. 
As técnicas cognitivo-comportamentais podem ser bastante eficazes na redução de muitos dos sintomas e deficiências comportamentais relacionados às doenças de fobias na infância. ${ }^{(13,26,23)}$

A Terapia Cognitivo-comportamental, mediante associação de suas diversas técnicas pode contribuir para o tratamento do Transtorno de Ansiedade Social, de modo que o paciente desenvolva as suas atividades sociais normalmente, e possa obter maior qualidade de vida. ${ }^{(8-17,19-26)}$

\section{CONCLUSÃO}

O Transtorno de Ansiedade Social pode causar sofrimento e sensação de incompetência no indivíduo, para vivenciar qualquer tipo de evento social, podendo prejudicálo tanto profissional, quanto socialmente. Verificou-se também que, a fobia social é muito prevalente na população geral, mas, muitos dos sujeitos portadores desse tipo de transtorno, não aceita a própria condição e a atribui a traços da personalidade.

Por serem as técnicas da TCC orientadas para a resolução de problemas, estas permitem ao indivíduo, desenvolver atividades sociais que restringiam as suas vidas. $O$ diagnóstico adequado, no entanto, permite à minimização, da maior parte, se não todos, os aspectos negativos que limitam a pessoa acometida por TAS. E, a sustentação de sessões ajuda a prevenir recaídas e desenvolver a confiança do paciente em si mesmo, ao passo em que o problema se torna infrequente, o sujeito passa a perceber o quanto tem capacidade para superar a questão.

A análise realizada sugere que as técnicas da TCC não podem ser aplicadas de modo isolado, devido à possibilidade de redução da sua eficácia. Bem como que, algumas técnicas são recomendadas para os pacientes, de modo geral, como o relaxamento e a exposição ao vivo, cujo objetivo é potencializar os resultados positivos ao realizar a reestruturação cognitiva.

$\mathrm{Na}$ atualidade, por ter aumentado o número de diferentes pessoas que buscam ajuda para tratar o TAS, este fenômeno passou a configurar um desafio para os psicólogos. Diante disso, cabe aos acadêmicos e, aos profissionais da área inteirarem-se das intervenções eficazes para este fim, para ser possível atuar com competência e, assim, auxiliar aos pacientes a alcançar melhores resultados.

Outubro, 2016:2(Edição Especial):41-53. 
Conclui-se que, a TCC apresenta boa eficácia na intervenção clínica e pode contribuir para o tratamento do TAS, através das suas diferentes técnicas, como exposição ao vivo, reestruturação cognitiva, relaxamento e treino das habilidades sociais. A TCC pode ser realizada em grupo, individual ou via internet, mas, sempre com o acompanhamento do terapeuta. Em suma, a TCC viabiliza a obtenção de resultados eficazes e duradouros para o tratamento de Transtorno de Ansiedade Social.

\section{REFERÊNCIAS}

1. American Psychiatric Association - APA. Manual Diagnóstico e Estatístico de Transtornos Mentais. DSM-5. Porto Alegre: Artmed; 2014.

2. D'El Rey GJF, PACINI CA. Tratamento da fobia social circunscrita por exposição ao vivo e reestruturação cognitiva. Revista Psiquiatria Clínica [Internet]. 2005 [2016 fev. 19];32(2):231-235. http://www.scielo.br/scielo.php?pid=S0101-60832005000400 005\&script=sci_arttext.

3. Cordioli AV, Knapp P. A terapia cognitivo-comportamental no tratamento dos transtornos mentais. Revista Psiquiatria Clínica [Internet]. 2008 [2015 set. 09];30(2):51-53.

://www.scielo.br/scielo.php?pid=S151644462008000600001\&script=sci_arttext .

4. Heimberg R. Cognitive behavioral therapy for social anxiety disorder: current status and future directions. Biol Psychiatry [Internet]. 2002 [2015 set. 09];51(.):101. http://www.ncbi.nlm.nih.gov/pubmed/11801235.

5. Gil AC. Como elaborar projeto de pesquisa. 4ª ed. São Paulo: Atlas; 2002.

6. Atallah AN. Revisão Sistemática e Metanálises, em: Evidências para melhores decisões clínicas.. Centro Cochrane do Brasil, Revisões Cochrane [Internet]. São Paulo; s. d. [2016 ago.11]. http://www.centrocochranedo brasil.org.br/cms/index.php?option=com_content\&View=article\&id=3\&ltemid=12

7. Lakatos E, Marconi M. A metodologia do trabalho científico. São Paulo: Atlas; 2010.

8. D'El Rey, GFJ et. al. A. Terapia cognitivo-comportamental de grupo no tratamento da fobia social generalizada. Psicol. Argum. 2007;25(50):305-311. 
9. D'El Rey, GFJ et. al. Tratamento cognitivo-comportamental de grupo na fobia social: resultados de 12 semanas Psiquiatria Clínica. 2008;35(50):79-83.

10. D’El Rey GJF, Beidel DC, Pacini CA. Tratamento da fobia social generalizada:comparação entre técnicas. Revista Brasileira de Terapia Comportamental e Cognitiva. 2006;7(1):001-012.

11. Diaz CP. Tratamiento cognitivo-conductual de un adolescente con trastorno de ansiedad generalizada. Revista de Psicología Clínica con Niños y Adolescentes. 2014;1(2):157-163.

12. Gusmão EES et.al. Contribuições da terapia cognitivo-comportamental para o tratamento da fobia social . Revista Brasileira de Terapia Cognitiva. 2013;9(2):118125.

13. Kahana SY. Cognitive Behavioral Treatment of Health-Related Anxiety in Youth: A Case Example. Cognitive and Behavioral Practice. 2005;12(.):290-300.

14. Antón D, Olivares-Olivares PJ, Amorós-Boix M. Detección e intervención conductualcognitiva en adultos-jóvenes con fobia social generalizada. Anales de Psicologia. 2012;28(1):19-27.

15. Mululo SCC, Menezes GB, Fontenelle L, et. al. Eficácia do tratamento cognitivo e/ou comportamental para o transtorno de ansiedade social. Revista Psiquiatria Clínica. 2009b;31(3):177-186.

16. Mululo SCC, Menezes GB, Fontenele L. Terapias cognitivo-comportamentais, terapias cognitivas e técnicas comportamentais para o transtorno de ansiedade social. Revista Psiquiatria Clínica [Internet]. 2009 [2015 set. 09];36(6):221-8. http://www.scielo.br/pdf/rpc/v36n6/v36n6a02.pdf.

17. Oliveira MIS. Intervenção cognitivo-comportamental em transtorno de ansiedade: relato de caso. Revista Brasileira de Terapia Cognitiva. 2011;7(1):30-34.

18. Viana CM. Tratamento da fobia social na abordagem cognitivo-comportamental. Revista de Psicologia. 2008;2(1):87-89.

19. Vidal Fernandes J, Ramos-Cejudo J, Cano-vindel A. Perspectivas cognitivas en el tratamiento de la fobia social: estudio de un caso clínico. Ansiedad y Estréz. 2008;14(23):289-303.

Outubro, 2016:2(Edição Especial):41-53. 
20. Levitan MN et.al. Diretrizes da Associação Médica Brasileira para o tratamento do transtorno de ansiedade social. Revista Brasileira de Psiquiatria. 2011;33(3):292-302.

21. Ito LM et. al. Phip. Terapia cognitivo-comportamental da fobia social . Revista Brasileira de Psiquiatria. 2008;30(2):96-101.

22. Antona CJ, García-López LJ. Repercusión de la exposición y reestructuración cognitiva sobre la fobia social. Revista Latino-americana de Psicologia. 2008;40(2):281-292.

23. Payne S, Bolton D, Perrin S. A pilot investigation of cognitive therapy for generalized anxiety disorder in children aged 7-17 years. Cogn Ther Res. 2011;35(.):171-178.

24. D'El Rey GJF, Abdallah H B. Intervenções cognitivas e comportamentais na fobia social. Revista Psiquiatria Clínica. 2006;(.):1-8.

25. D'El Rey GJF, Paccini CA. Terapia cognitivo-comportamental da fobia social: modelos e técnicas. Psicologia Estud. 2006;11(2):269-275.

26. Petersen CS. Evidências de efetividade e procedimentos básicos para Terapia Cognitivo-Comportamental para crianças com transtornos de ansiedade.. Revista Brasileira de Psiquiatria. 2011;13(1):39-50.

27. Gallart GDV, Ferrero MJ, Furlan L. Comorbilidades de laansiedad frente losexámenes em estudiantes universitários [Congresso]. Buenos Aires: Facultad de Psicología Universidad de Buenos Aires;2013.

28. D’El Rey GJF, Pollack P N. Ansiedade grave: características, prevalência de sintomas e procura de tratamento profissional em uma amostra de pessoas. Arq. Ciência e Saúde UNIPAR. 6(2): 129-132; 2002

29. Crawley SA et. al. Brief Cognitive-Behavioral Therapy for Anxious Youth: Feasibility and Initial. Outcomes. Cognitive and Behavioral Practice. Outcomes. 2013;20(2):123133.

30. Castro C G S O. Estudos de utilização de medicamentos. Noções Básicas. Rio de Janeiro. 2000; 1(1):15-25.

31. Gonçalves W J, Silva A L. Estudos de utilização de medicamentos. Noções Básicas. Perfil da comercialização da quetiapina e suas implicações clínicas no tratamento do

Outubro, 2016:2(Edição Especial):41-53. 


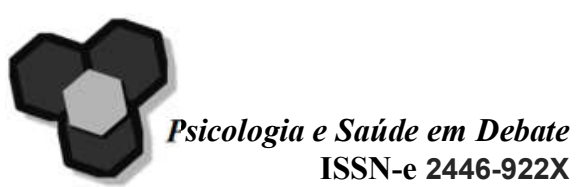

transtorno do humor bipolar [Internet]. 2016 [2016 jun. 29];2(1):41. https://psicodebate.files.wordpress.com/2016/05/3-gonc3a7alves-e-silva-2016.pdf.

Outubro, 2016:2(Edição Especial):41-53. 